\title{
Use of Gas Sensors and FOBT for the Early Detection of Colorectal Cancer ${ }^{\dagger}$
}

\author{
Giulia Zonta 1,* , Gabriele Anania ${ }^{1}$, Aldo de Togni ${ }^{2}$, Andrea Gaiardo ${ }^{1}$, Sandro Gherardi ${ }^{3}$, \\ Alessio Giberti ${ }^{4}$, Vincenzo Guidi ${ }^{1}$, Nicolò Landini ${ }^{1,3}$, Caterina Palmonari ${ }^{1,2}$, Luciano Ricci ${ }^{3}$ and \\ Cesare Malagù ${ }^{1}$ \\ 1 Department of Physics and Earth Sciences, University of Ferrara, Via Savonarola, 9-44121 Ferrara, Italy; \\ ang@unife.it (G.A.); andrea.gaiardo@unife.it (A.G.); guidi@fe.infn.it (V.G.); nicolo.landini@unife.it (N.L.); \\ c.palmonari@ausl.fe.it (C.P.); malagu@fe.infn.it (C.M.) \\ 2 Department of Public Health (AUSL), UO Igiene Pubblica, Via Fausto Beretta, 7-44121 Ferrara, Italy; \\ a.detogni@ausl.fe.it \\ 3 SCENT S.r.l., Via Quadrifoglio, 11-44124 Ferrara, Italy; gherardi@fe.infn.it (S.G.); \\ dr.lucianoricci@gmail.com (L.R.) \\ 4 MIST E-R S.c.r.l., Via P. Gobetti 101, 40129 Bologna, Italy; giberti@fe.infn.it \\ * Correspondence: giulia.zonta@unife.it; Tel.: +39-0532-974286 \\ + Presented at the Eurosensors 2017 Conference, Paris, France, 3-6 September 2017.
}

Published: 31 August 2017

\begin{abstract}
Among the major challenges of medicine today there are screening and early detection of tumors (since the adenoma stage) in order to prevent their degeneration into malignant cancer and/or metastases. In particular, the colorectal cancer shows a high curability rate, up to $90 \%$, if identified when in its benign stage. The Protocol discussed here is proposed to implement the clinical validation of a device consisting of an array of chemoresistive gas sensors made of semiconductor materials, able of identifying the difference between fecal exhalation of healthy subjects and of subjects suffering from high-risk colorectal polyps or tumors. The tests are compared to the results of fecal occult blood test and colonoscopy as a gold standard.
\end{abstract}

Keywords: gas sensors; colorectal cancer; screening

\section{Introduction}

In the present work, the purpose is to discriminate healthy subjects from subjects affected by colorectal cancer (CRC) since the stage of advanced adenoma, by means of a non-invasive method. Currently, the test accepted in Italy and other countries (e.g., UK, France) is the fecal occult blood test (FOBT), performed on people between 50 and 69 years of age. Despite being a valid tool for detecting blood presence in the stool, FOBT shows a huge number of false positives [1,2]. Therefore, the importance of an additional screening system lies in the decrease of false positive rate, to reduce the number of non-operative colonoscopies, risky for elderly and debilitated patients. Previous works by our team have shown that chemoresistive sensors are capable of detecting tumor biomarkers in very low concentrations (tenths of ppb) [3,4]. The technique presented here employs a patented device [5] named SCENT A1, made of a core of five chemoresistive sensors. SCENT A1 can identify cancer by the odor of fecal exhalations. The difference between fecal samples of healthy subjects and of CRCaffected patients is due to two main processes: (i) the peroxidation of tissues affected by cancerous and precancerous lesions; (ii) the alteration of the metabolic activity of cells. Here are shown both the data coming from a feasibility study to set-up the sensor array and the data obtained by a clinical validation Protocol, started in May 2015 and still on-going. 


\section{Materials and Methods}

SCENT A1 is equipped with a pneumatic system that allows sending an environmental airflow, contaminated by fecal fumes, to the sensing unit. The sensors vary their resistance in contact with the gas and can distinguish different mixtures. Each sensor is composed of a film of nanostructured semiconductor material [6,7].

\subsection{Feasibility Study}

The feasibility study has been performed in order to find the best five-sensor combination, in collaboration with the Department of Morphology, Surgery and Experimental Medicine of the University of Ferrara (UNIFE). The first data analysis method chosen is the principal component analysis (PCA), through which it is possible to visualize each sample tested as a point in a Cartesian graph and to identify two different ellipsoidal regions for healthy and CRC-affected individuals. The smaller the overlap between the two areas and the greater the precision of the test. The chosen combination of sensors has been calibrated, after testing 19 different materials (both metal-oxides and -sulfides), so as to maximize the difference between the two sample types (feces of healthy subjects and subjects with CRC, taken during surgery), thanks to an activity previously carried out in the laboratory on 170 samples [8,9]. The PCA related to one of the most discriminating arrays is shown in Figure 1. The green dots represent 18 control samples while the blue ones are 10 samples from subjects with CRC. The areas identify the two populations and the overlap is minimal. This is indicative of a high degree of distinction. The ROC curve, in Figure 2, was calculated by considering all the sample tested with all the sensor arrays tried. The curve shows both a sensitivity and a specificity of $95 \%$ and also the accuracy of the test has been estimated as $95 \%$. The materials chosen after this study were both single metal-oxides and mixtures of them (i.e., indium, tin, titanium and vanadium oxide), fired at a temperature of $650^{\circ} \mathrm{C}$.

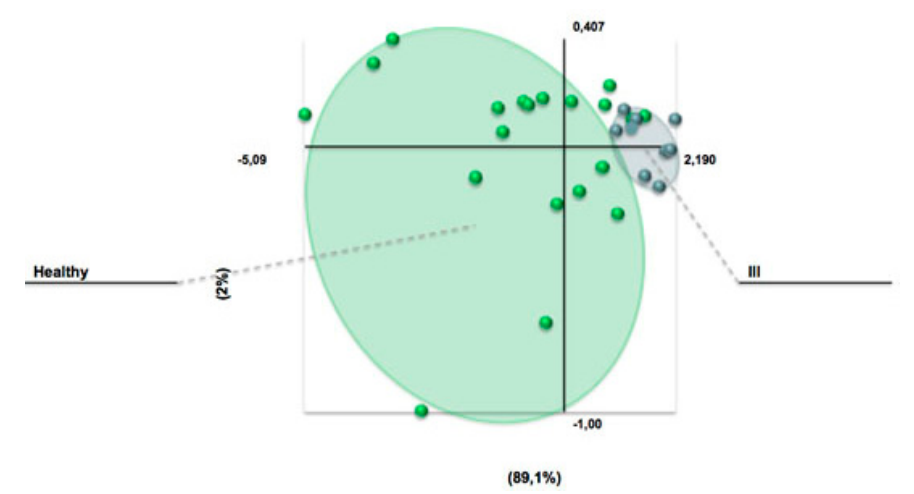

Figure 1. PCA related to the chosen array. Here are shown 18 control samples (green dots) and 10 samples from subjects with CCR (blue dots). The areas represent the two populations.
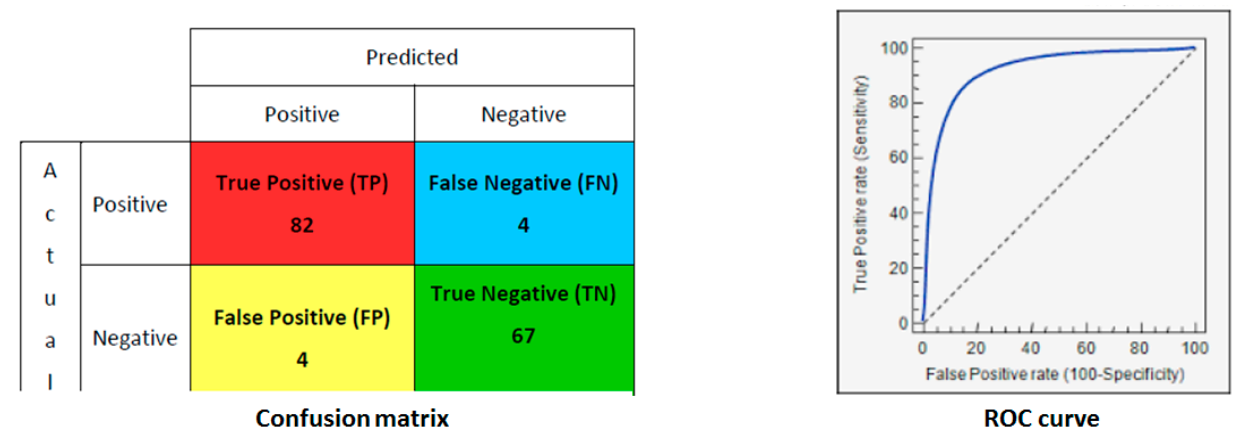

Figure 2. Confusion matrix (on the left) and ROC curve (on the right) made with 157 samples with different five-sensor combinations of 19 materials. True Positive Rate $(\mathrm{TPR})=$ sensitivity $=95 \%$, True Negative Rate $($ TNR $)=$ specificity $=95 \%$. 


\subsection{Clinical Validation}

Since May 2016, following the approval by the Ethics Committee, it has been started a Protocol for the clinical validation of the device. To all of screening users in Ferrara, who resulted positive to FOBT, is proposed to participate in the experimental study SCENT A1 before undergoing colonoscopy investigation (chosen as the gold standard). Over $90 \%$ of patients have adhered to the study. Colonoscopy results are fundamental to obtain a sufficient statistic to calibrate the device and to set a priori the accuracy of the method. In addition to SCENT A1 tests, a volunteer physician, engaged in the interview with the patients, developed an assessment based on questions submitted to the subjects. These questions concern familiarity, symptoms and other inflammatory diseases suffered from patients. A comparison between evaluations obtained through this medical consultation and colonoscopy outcomes may provide added value to the fundamental importance of a screening test. The sensitivity and specificity of an expert eye are respectively of $11 \%$ and $96 \%$, evaluated on a sample of 131 subjects, of which we had both the doctor's overthrow and the colonoscopy outcome. If only the average/high risk adenomas and cancers are considered as positives the sensitivity results null. This qualitative data gives an idea of the crucial importance of a preventive screening for CRC to avoid a so large number false negatives. So far, the sensor responses of a total of 234 fecal samples have been measured with SCENT A1 during the Protocol. The precision of FOBT test estimated from the data that have already a colonoscopy result (174) is only of $28 \%$. This means that the number of false positives is very high and this involves a large number of non-operative colonoscopies, submitting healthy patients to an unnecessary risk of intestinal perforation. The addition of a complementary test, i.e., SCENT A1, that relies on a gaseous marker totally different from occult blood, should be fundamental to reduce false positive rate.

\section{Results and Discussion}

Since now, 86 of the samples measured with SCENT A1 have been compared with the gold standard. They have been analyzed by means of PCA and Support Vector Machine (SVM) techniques, dividing the three class of subjects:

- $\quad$ cancer and high-risk adenomas (CRC+HR);

- low-risk adenomas (LR);

- $\quad$ healthy subjects (HS).

Results analysis are summarized in Table 1. It appears evident from these data, that all the CRC+HR are correctly identified so as the largest part of HS (97\%) and LR $(90 \%)$. The error on LR is not so problematic, in fact, for low risk adenomas, the risk for degeneration is negligible. For this reason, classifying subjects with low risk adenomas as healthy and re-screening them after two years, it could be a way of reducing colonoscopies to the benefit of the patients' health.

Table 1. Results obtained by analyzing a part of the responses of the samples measured within the Protocol that compares FOBT with SCENT A1 test and colonoscopy results. This data have been extrapolated by PCA treatment and SVM.

\begin{tabular}{lcccc}
\hline & & \multicolumn{3}{c}{ Predicted } \\
\cline { 3 - 5 } & & HS & LR & CRC + HR \\
\hline \multirow{3}{*}{ Actual } & HS & 58 & 1 & 1 \\
& LR & 2 & 18 & 0 \\
& CRC + HR & 0 & 0 & 6 \\
\hline
\end{tabular}

The clinical validation will continue for two years.

In Table 2, it is reported a comparison of the data obtained so far from the present study. These data will be progressively updated during the continuation of the tests. From September 2017, the Protocol will be extended to the Delta Hospital in Lagosanto (Italy), with the aim of increasing the statistics on true positives. 
Table 2. Comparison of FOBT with the SCENT A1 data of feasibility study and patients' health evaluation during the interview. Samples are all from patients resulted positive to FOBT, so it is possible to estimate only the precision for this test. Data coming from the clinical validation of SCENT A1 are not shown due to the fact that they are split in three categories.

\begin{tabular}{cccc}
\hline & FOBT & SCENT A1 & Interview \\
\hline Sensitivity (TPR) & - & $95 \%$ & $11 \%$ \\
Specificity (TNR) & - & $95 \%$ & $96 \%$ \\
Accuracy & - & $95 \%$ & $72 \%$ \\
Precision & $28 \%$ & $95 \%$ & $44 \%$ \\
\hline
\end{tabular}

Conflicts of Interest: The authors declare no conflicts of interest

\section{References}

1. Elsafi, S.H.; Alqahtani, N.I.; Zakary, N.Y.; Al Zahrani, E.M. The sensitivity, specificity, predictive values, and likelihood ratios of fecal occult blood test for the detection of colorectal cancer in hospital settings. Clin. Exp. Gastroenterol. 2015, 8, 279-284.

2. Shin, A.; Son Choi, K.; Kwan Jun, J.; Keun Noh, D.; Suh, M.; Jung, K.W.; Chang Kim, B.; Hwan Oh, J.; Park, E.C. Validity of Fecal Occult Blood Test in the National Cancer Screening Program, Korea. PLoS ONE 2013, 8, e79292.

3. Malagù, C.; Fabbri, B.; Gherardi, S.; Giberti, A.; Guidi, V.; Landini, N.; Zonta, G. Chemoresistive gas sensors for detection of colorectal cancer biomarkers. Sensors 2014, 14, 18982-18992.

4. Zonta, G.; Anania, G.; Fabbri, B.; Gaiardo, A.; Gherardi, S.; Giberti, A.; Guidi, V.; Landini, N.; Malagù, C. Detection of colorectal cancer biomarkers in the presence of interfering gases. Sens. Actuators B 2015, 218, 289-295.

5. SCENT A1. Patent and Intellectual Property of SCENT S.r.l., Patent Number: WO2016063148, published in 28 April 2016. European internationalization in progress.

6. Gaiardo, A., Fabbri, B., Guidi, V., Bellutti, P., Giberti, A., Gherardi, S., Vanzetti, L., Malagù, C., Zonta, G. Metal sulfides as sensing materials for chemoresistive gas sensors Sensors (Switzerland) 2016, 16 (3).

7. Gaiardo, A., Fabbri, B., Giberti, A., Guidi, V., Bellutti, P., Malagù, C., Valt, M., Pepponi, G., Gherardi, S., Zonta, G., Martucci, A., Sturaro, M., Landini, N.

8. $\mathrm{ZnO}$ and $\mathrm{Au} / \mathrm{ZnO}$ thin films: Room-temperature chemoresistive properties for gas sensing applications Sensors and Actuators, B: Chemical 2016, 237, pp. 1085-1094.

9. Landini, N.; Zonta, G.; Malagù, C. Detection of Tumor Markers on Feces with Nanostructured Sensors; Scholars' Press, Saarbrucken, Germany: 2015; ISBN-13:978-3-639-76538-0.

10. Zonta, G.; Anania, G.; Fabbri, B.; Gaiardo, A.; Gherardi, S.; Giberti, A.; Landini, N.; Malagù, C.; Scagliarini, L.; Guidi, V. Preventive screening of colorectal cancer with a device based on chemoresistive sensors Sens. Actuators B 2016, 238, 1098-1110.

(C) 2017 by the authors. Licensee MDPI, Basel, Switzerland. This article is an open access article distributed under the terms and conditions of the Creative Commons Attribution (CC BY) license (http://creativecommons.org/licenses/by/4.0/). 